\title{
Effects of end plates on performance of a small straight-bladed vertical axis wind turbine
}

\author{
Seiji YAMADA*, Tomohiro TAMURA* and Shinsuke MOCHIZUKI** \\ * Yamaguchi Prefectural Industrial Technology Institute, 4-1-1 Asutopia, \\ Ube City, Yamaguchi, 755-0195, Japan \\ E-mail: seiji@iti-yamaguchi.or.jp \\ ** Graduate School of Science and Engineering, Yamaguchi University, 2-16-1 Tokiwadai, \\ Ube City, Yamaguchi, 755-8611, Japan
}

Received: 25 August 2017; Revised: 30 October 2017; Accepted: 3 December 2017

\begin{abstract}
The time mean characteristics and the torque variation of the straight-bladed vertical axis wind turbine attached with various end plates were examined in wind tunnel experiments, and they were associated with the effect of the wing tip shape on the performance of the wind turbine. In the low tip speed ratio range, the time mean torque and the time mean power coefficients are reduced by attaching end plates. In the high tip speed ratio range, the time mean torque and the time mean power coefficients are increased by attaching end plates except for certain condition. Their maximum increase takes place at the condition that end plates are attached horizontally toward the side of the rotating axis. The effect of end plates was discussed by comparing the variation in the phase averaged torque coefficient. For each condition at the low tip speed ratio and the high tip speed ratio, the phase averaging analysis is able to specify the particular phase concerned to the significant change of the time mean torque coefficient and give the physical interpretation of the phenomenon.
\end{abstract}

Keywords : Straight-bladed vertical axis wind turbine, End plate, Performance, Mean characteristics, Torque variation, Wind tunnel experiment

\section{Introduction}

Expectations for the use of renewable energy are increasing to solve the global energy and environmental problems associated with $\mathrm{CO}_{2}$ emission. The present experimental study focuses on a small wind turbine which has possibility to introduce renewable energy easily in suburbs and others.

A straight-bladed vertical axis wind turbine (This is called commonly Straight Darrieus Turbine) is one of the reliable wind turbines, since it is free from the directional control for variable oriented natural wind, produces low noise for the lower rotational speed, and has simple blade structure (Ushiyama, I., 2002, Mizuno, A., 2002).

Specifications of blades such as wing forms of blades, blade tip shapes, solidities affect the mean characteristics of the wind turbine, so many researches on them have been done so far (e.g. Asao, T., et al., 2009). The blade tip shape is one of particularly important specifications, since the tip vortex generated at the blade tip in the three-dimensional blade has a great effect on the blade performance (Furuya, Y., 1973).

About effects of blade tip shape on the performance of the blade, there are literatures on additional nonlinear lift by wing tip vortex for the low aspect ratio wing (Mueller, T. J., 2003), on the effectiveness of the winglet or the wing tip sail for the reduction of the induced drag of the wing (Murotsu, Y. edited, 2006), on aerodynamic gain and structural loss by varying the setting angle of the end plate (Kubota, H. et al., 1983) and so on. However, these are supposed to be the wing of the aircraft and concern only the static characteristics. On the other hand, in the case of a vertical axis type wind turbine, the relative wind velocity and the angle of attack relative vary considerably depending on the azimuth angle of the blade, and it also becomes higher angle of attack above the stall angle not experienced on a wing of the aircraft. 
Regarding the vertical axis wind turbine blades, there are some studies on the development of the wing tip vortex and its strength for different wing tip shapes (Hofemann, C., et al., 2008) and on the effect of wing tip shapes of the blades on mean characteristics (Nishizawa, Y., et al., 2009). The net rotational torque of this wind turbine due to the force acting on the blade from the wind varies (Yamada, S., et al., 2011), since the relative wind velocity and the angle of attack relative vary with the azimuth angle of the blade. The torque variation with the azimuth angle affects mean characteristics (torque and power characteristics) of the wind turbine (Yamada, S., et al., 2011) are essential properties in order to understand the performance of this wind turbine.

Therefore, we investigate the influence of the blade tip shape on the performance of the straight-bladed vertical axis wind turbine, mean characteristics and the torque variation characteristics with various types of end plates in wind tunnel experiments and try to find physical relations of them.

\section{Nomenclature}

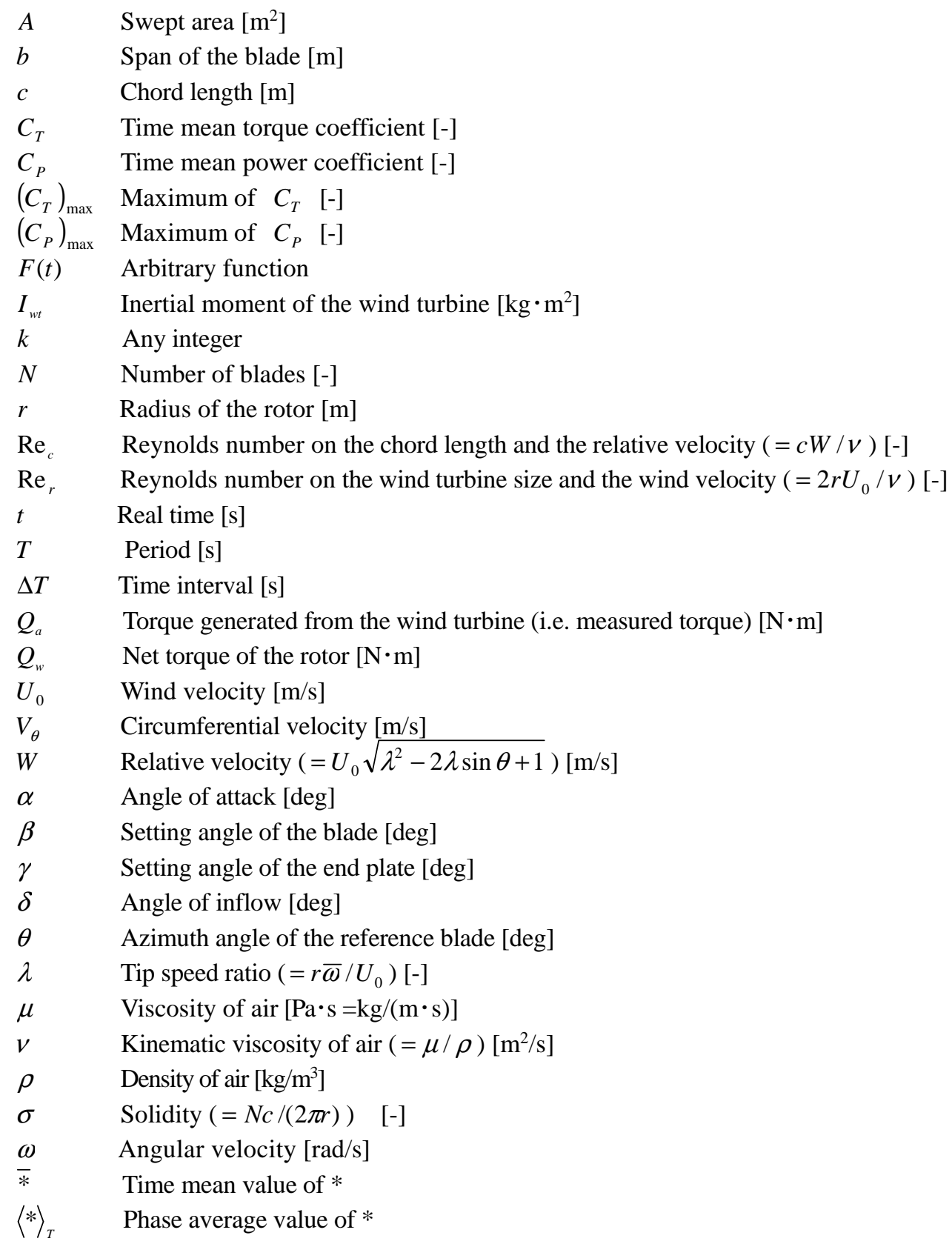




\section{Dynamical model of the wind turbine}

The dynamic model of the wind turbine is shown in Fig. 1. The dynamic equation of the wind turbine is

$$
I_{w t} \frac{d \omega}{d t}=Q_{w}-Q_{a}
$$

where $Q_{w}$ is the net torque $[\mathrm{N} \cdot \mathrm{m}], Q_{a}$ is the torque generated from the wind turbine(i.e. measured torque)[N·m], $\omega$ is the angular velocity of the rotor[rad/sec], and $I_{w t}$ is the inertial moment of the wind turbine $\left[\mathrm{kg} \cdot \mathrm{m}^{2}\right]$. The net torque of the rotor by the wind is calculated by

$$
Q_{w}=Q_{a}+I_{w t} \frac{d \omega}{d t}
$$

The wind turbine was connected to the motor via the torque converter, and the torque of the rotor is measured under constant rotational speed. If the inertial term is ideally neglected, namely $Q_{w}=Q_{a}$. However, actually $\omega$ has certain variation due to variation in $Q_{w}$ (Yamada, S., et al., 2011). In the present experiment, the inertial term is remained in order to calculate the net torque variation in a rotation. On the mechanical friction effect in measured torque, the friction torque, which is measured at each rotational speed without the wind turbine, is added the measured torque.

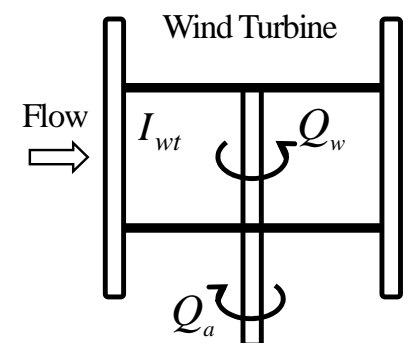

Fig. 1 Dynamical model of the wind turbine.

\section{Experimental Techniques}

The experimental apparatus and the coordinate system are shown in Fig.2. $U_{0}[\mathrm{~m} / \mathrm{s}]$ is the wind velocity and $\theta$ [deg] is the azimuth angle of the reference blade. The wind turbine is located in open space in front of the exit of the wind tunnel test section with the $1000 \mathrm{mmW} \times 1000 \mathrm{mmH}$ rectangular cross section. In this case, the influence of the blockage effect is considerably small as compared with the case where the wind turbine is located in the closed space inside the measurement cave (For example, the correction amount of the open wind tunnel is 1/5 or less of the closed wind tunnel (Hashidate, M. and Ishida, Y., 1991)). Therefor it is not necessary to consider the blockage effect of the wind turbine on the wind tunnel test section. While effect of non-uniformity of the velocity is supposed, but from the wind velocity profile

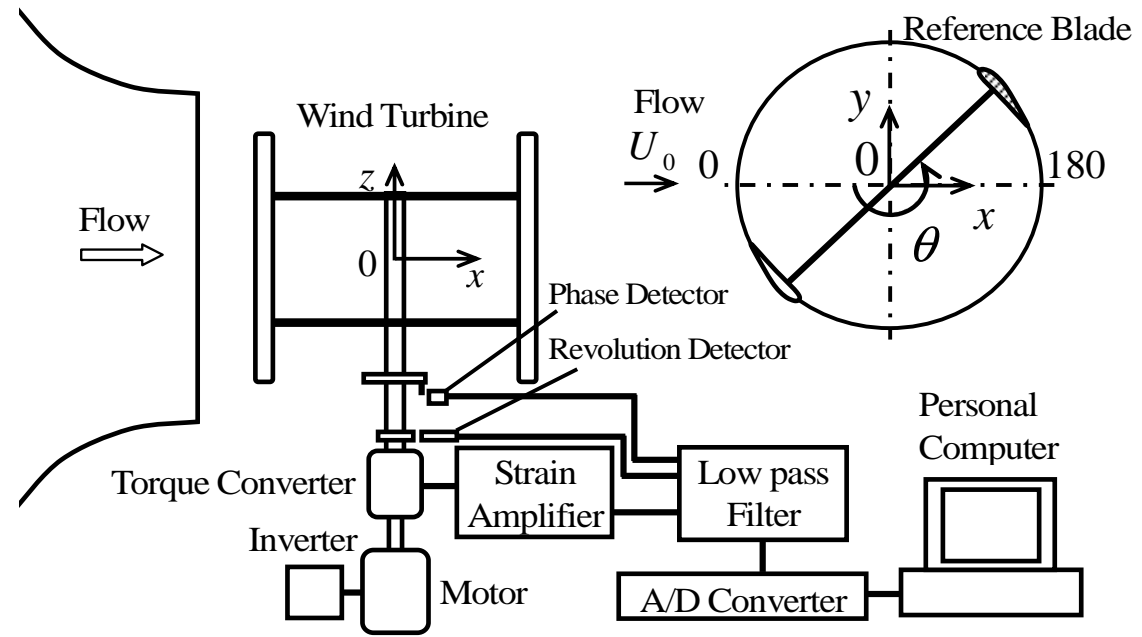

Fig. 2 The experimental apparatus and the coordinate system. 
measurement at the setting position of the wind turbine, the wind turbine was within the potential core of the flow produced by the wind tunnel, so that it is not necessary to consider the effect of non-uniformity of the velocity. In detail, the wind velocity profile immediately after the wind tunnel nozzle exit is within $3 \%$ over the entire cross section of $1,000 \mathrm{~mm} \times 1,000 \mathrm{~mm}$. In addition, at $1500 \mathrm{~mm}$ downstream from the wind tunnel outlet on the downstream side of the wind turbine, the potential core is confirmed in the region of the wind tunnel center part of $800 \mathrm{~mm} \times 800 \mathrm{~mm}$. The wind turbine was located on the center of the wind tunnel, and the distance of between the wind tunnel nozzle exit and the center of rotation of the wind turbine is $450 \mathrm{~mm}$. The origin of coordinate is located the center of the wind turbine height on the rotation axis of the rotor. The Cartesian coordinate is employed; the x-axis is parallel to the main flow direction and the $\mathrm{z}$-axis is vertical direction. The position of $0[\mathrm{deg}]$ for the azimuth angle $\theta$ is located at the most upstream against the main stream, and positive direction is clockwise to the $\mathrm{z}$-axis direction. The velocity of the flow approaching to the wind turbine is measured by a pitot tube located at just exit of the wind tunnel. The wind turbine is connected to the motor via the torque converter and controlled in a constant rotational speed by the inverter. The torque of the rotor, the angle of rotation, and the phase detection signal of the reference blade are measured simultaneously, and the time series data converted from the analog signal to the digital signal at $10 \mathrm{kHz}$ for 30 seconds are stored in a computer to calculate various statistics. The sampling interval of the azimuth angle is the maximum $0.216^{\circ}$ at the rotational speed of $360 \mathrm{rpm}$. The time mean torque $\bar{Q}_{w}$, the time mean angular velocity $\bar{\omega}$, the phase average measured torque $\left\langle Q_{a}\right\rangle_{T}$ and the phase average angular acceleration $\langle d \omega / d t\rangle_{T}$ are calculated from the time series data analysis. Here, $\langle *\rangle_{T}$ represents the phase average to be described later. These are actually obtained using time series data of 30 to180 cycles. With respect to the estimated value of phase average torque, when the number of phase averaging period is 30 , the standard deviation of the average value is $18.3 \%$ of the population standard deviation. Assuming that the influence of noise due to the vibration of the equipment etc. increases as the rotational speed increases, the number of phase averaging period at the maximum rotational speed of $360 \mathrm{rpm}$ is 180 . The net torque of the rotor by the wind is calculated by equation (2) using the phase average measured torque $\left\langle Q_{a}\right\rangle_{T}$ and the phase average angular acceleration $\langle d \omega / d t\rangle_{T}$. Conventional time mean and phase average of arbitrary function $F(t)$ are defined as

$$
\begin{aligned}
& \bar{F}(t)=\lim _{\Delta T \rightarrow \infty} \frac{1}{\Delta T} \int_{0}^{\Delta T} F(t) d t \\
& \langle F(t)\rangle_{T}=\lim _{N \rightarrow \infty} \frac{1}{N} \sum_{k=0}^{N-1} F(t+k T), \quad(0 \leq t \leq T, \quad T \text { is the period })
\end{aligned}
$$

The coefficients for net time mean torque, time mean power and phase average torque by wind are defined as follows;

$$
C_{T}=\frac{\bar{Q}_{w}}{1 / 2 \rho U_{0}^{2} A r} \quad(5), \quad C_{P}=\frac{\overline{Q_{w}} \bar{\omega}}{1 / 2 \rho U_{0}^{3} A} \quad\left\langle C_{T}\right\rangle_{T}=\frac{\left\langle Q_{w}\right\rangle_{T}}{1 / 2 \rho U_{0}{ }^{2} A r}
$$

where $\rho$ is air density $\left[\mathrm{kg} / \mathrm{m}^{3}\right], A$ is swept area $\left[\mathrm{m}^{2}\right]$, and $r$ is rotor radius $[\mathrm{m}]$. In this experiment, $A$ is swept area including end plates.

The test wind turbine has rotor radius of $r=0.3015[\mathrm{~m}]$, blade span of $b=0.6[\mathrm{~m}]$, chord length of $c=150[\mathrm{~mm}]$, number of blades of $N=2$ or 1 (solidity $\sigma=N c / 2 \pi r=0.16$ at $N=2$ ). $r$ is the distance between the leading edge of the airfoil and the center of rotation of the wind turbine. The setting angle of the blade $\beta$ is the angle between the tangent of the wind turbine rotation circumference passing through the leading edge and the chord line. It is the angle which the maximum power coefficient is the largest. $\beta=14.18$ [deg] in all blade tip shapes of this experiment.

The blades are attached to arms at the distance of $c$ from the tip of the blades. The wing section of the blade is NACA0020 designed according to NACA 4 digit wing section. The plane shape of the blade is rectangle. The blade material is expanded polystyrene. In order to investigate effects of end plates, semi-circular end plates having a thickness of $1.0 \mathrm{~mm}$ and a radius of $c / 2$ were attached to both ends of the blade at different angles. Figure 3 shows the state of attachment of end plates to the blade in a two-blade wind turbine. In the case of a single-blade wind turbine, in the two-blade wind turbine shown in Fig. 3, only one blade is detached with the setting arms intact. The setting angle $\gamma$ of the end plate is set to $\gamma>0$ on the opposite side to the rotating shaft with respect to the blade width direction. It is set to $\gamma= \pm 45^{\circ}$ and $\pm 90^{\circ}$. In addition to the case without the end plate, the total of 5 conditions are examined. Experiments were made at the wind velocity $U_{0}=6[\mathrm{~m} / \mathrm{s}]$. Then the Reynolds number $\operatorname{Re}_{r}=2 r U_{0} / v$ is $2.4 \times 10^{5}$. For 
the other Reynolds number, when the tip speed ratio $\lambda\left(=r \bar{\omega} / U_{0}\right)=1.34$, it is $\operatorname{Re}_{c}=W c / v=2.0 \times 10^{4}$ to $1.4 \times 10^{5}$. Where $W$ is the relative velocity for the blade and $v$ is kinematic viscosity of air. The performance of a small straight-bladed vertical axis wind turbine depends on the Reynolds number. For any given shape parameters the chord length/rotor radius $c / r$, aspect ratio $b /(2 r)$ and the solidity $\sigma$ on specifics of the wind turbine having the same wing section, the mean torque and power coefficients can be well represented by the logarithmic functions of $\operatorname{Re}_{r}$ and quadratic or cubic function of the tip speed ratio $\lambda$ (Yamada, S. et al., 2016).

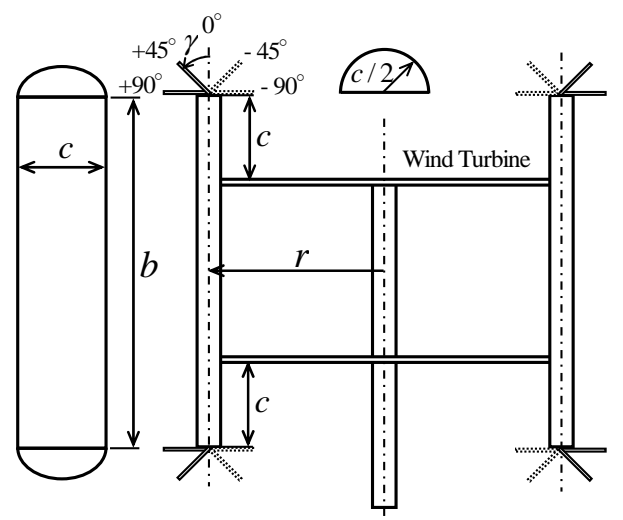

Fig.3 Attached End plates to the blade.

\section{Experimental Results and Discussion}

\subsection{Mean characteristics of the wind turbine}

Figure 4 shows the time mean torque and power characteristics of the wind turbine with two blades having four types of end plates with different setting angles and without the end plate. The horizontal axis is the time mean tip speed ratio $\lambda\left(=r \bar{\omega} / U_{0}\right)$, and the vertical axis is the time mean torque coefficient $C_{T}$ (upper side) and the time mean power coefficient $C_{P}$ (lower side). $C_{T}$ of the wind turbine without the end plate is the largest in low tip speed ratio range of $\lambda<0.87 . C_{T}$ is decreased by attaching end plates. $C_{T}$ is increased by attaching end plates except for $\gamma=+90^{\circ}$

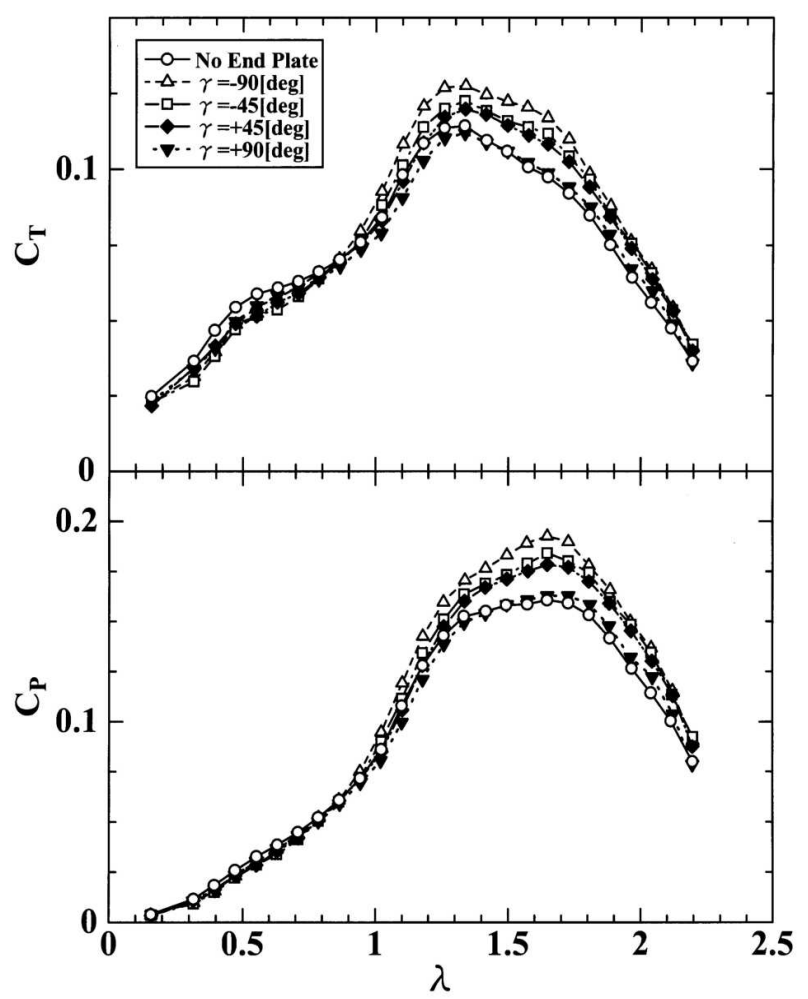

Fig.4 The effect of end plates on the time mean torque and the time mean power.

The time mean torque coefficient $C_{T}$ (upper side). The time mean power coefficient $C_{P}$ (lower side). 
in high tip speed ratio range of $\lambda>1$.18. In all conditions, $C_{T}$ takes the maximum value $\left(C_{T}\right)_{\max }$ at $\lambda \cong 1.34$, and the largest value is $\left(C_{T}\right)_{\max }=0.13$ at $\gamma=-90^{\circ} .\left(C_{T}\right)_{\max }$ takes almost the same value for $\gamma=-45^{\circ}$ and $\gamma=+45^{\circ}$, and $\left(C_{T}\right)_{\max }$ at $\gamma=+90^{\circ}$ and $\left(C_{T}\right)_{\max }$ without the end plate are comparable in magnitude. In detail, the order of the magnitude of the value is $\gamma=-90^{\circ}, \gamma=-45^{\circ}, \gamma=+45^{\circ}$, without the end plate, $\gamma=+90^{\circ}$. $C_{T}$ of $\gamma=-45^{\circ}$ and $\gamma=+45^{\circ}$ show relatively similar tendency of the variation over the whole range of $\lambda$. In the range of $\lambda$ larger than $\lambda$ where $\left(C_{T}\right)_{\max }$ takes place, the variations of $C_{T}$ at $\gamma=+90^{\circ}$ and without the end plate are almost similar.

Reflecting the time mean torque characteristic, in the low tip speed ratio range of $\lambda<0.87, C_{P}$ of the wind turbine without the end plate is the largest, that is, $C_{P}$ is decreased by attaching end plates. $C_{P}$ is increased by attaching end plates except for $\gamma=+90^{\circ}$ in high tip speed ratio range of $\lambda>1.18$. In all conditions, $C_{P}$ takes the maximum value $\left(C_{P}\right)_{\max }$ at $\lambda \cong 1.65$, and the largest value is $\left(C_{P}\right)_{\max }=0.19$ at $\gamma=-90^{\circ} .\left(C_{P}\right)_{\max }$ at $\gamma=+90^{\circ}$ and without the end plate are comparable in magnitude. In detail, the order of the magnitude of the value is $\gamma=-90^{\circ}$, $\gamma=-45^{\circ}, \gamma=+45^{\circ}, \gamma=+90^{\circ}$, without the end plate. $C_{P}$ at $\gamma=-45^{\circ}$ and $\gamma=+45^{\circ}$, and $C_{P}$ at $\gamma=+90^{\circ}$ and without the end plate show similar tendency of variation over the whole range of $\lambda$.

From the mentioned above experimental results, $C_{T}$ and $C_{P}$ are decreased by attaching end plates in low tip speed ratio range of $\lambda<0.87$. On the other hand, $C_{T}$ and $C_{P}$ are increased by attaching end plates except for $\gamma=+90^{\circ}$ in high tip speed ratio range of $\lambda>1.18 .\left(C_{T}\right)_{\max }$ and $\left(C_{P}\right)_{\max }$ are largest at $\gamma=-90^{\circ}$.

\subsection{Characteristics of torque variation}

\subsubsection{Torque variation of the two-blade turbine}

Figure 5 (a) and (b) show torque variation as a function of the azimuth angle $\theta$ of the wind turbine with two blades having four types of end plates with four different setting angles and without the end plate. The two blades are set every $180^{\circ}$, and the torque variations between $\theta=0^{\circ}$ to $180^{\circ}$ and $\theta=180^{\circ}$ to $360^{\circ}$ are relatively well agreement. In the figures, the phase of the torque variation of $\theta=180^{\circ}$ to $360^{\circ}$ is shifted by $180^{\circ}$ so as to correspond to the torque variation of $\theta=0^{\circ}$ to $180^{\circ}$, and these variation are averaged for each phase, variations in the range of $\theta=0^{\circ}$ to $180^{\circ}$ are shown in the figures. The vertical axis is the net phase average torque coefficient $\left\langle C_{T}\right\rangle_{T}$ by wind. Figure 6 (a) and (b) show the maximum and the minimum phase average torque coefficients of these five wind turbines with two blades. As a representative, $\lambda=0.63$ in the low tip speed ratio region and $\lambda=1.34$ where $C_{T}$ takes the maximum are shown. As reference, Fig. 7 (a) and (b) show the angle of attack $\alpha$ and relative velocity $W / U_{0}$ of each phase with respect to the reference blade, respectively. They are obtained assuming that the wind turbine rotates in the uniform flow $U_{0}$ at the circumferential velocity $V_{\theta}=r \omega$, and there is no influence on the flow field by other blades. $\alpha$ and $W / U_{0}$ are represented by the following expressions.

$$
\begin{aligned}
& \alpha=\delta+\beta \\
& \delta=\tan ^{-1}\left(\frac{-\cos \theta}{\lambda-\sin \theta}\right) \\
& W / U_{0}=\sqrt{\lambda^{2}-2 \lambda \sin \theta+1}
\end{aligned}
$$

where $\delta$ is angle of inflow (The angle formed by the relative wind velocity with respect to the tangential direction of the rotational circumference).

For $\lambda=0.63,\left\langle C_{T}\right\rangle_{T}$ for $\gamma<0$ takes the maximum value $\left(\left\langle C_{T}\right\rangle_{T}\right)_{\max }$ in all conditions when the reference blade is in the range of $\theta=161^{\circ}$ to $167^{\circ}$, and the other blade is in the range of $\theta=341^{\circ}$ to $347^{\circ}$ near the most upstream $\left(\theta=0^{\circ}\right)$. $\left(\left\langle C_{T}\right\rangle_{T}\right)_{\max }$ is increased by attaching end plates, and is the largest at $\gamma=-90^{\circ} .\left\langle C_{T}\right\rangle_{T}$ takes the minimum value $\left(\left\langle C_{T}\right\rangle_{T}\right)_{\min }$ in the range of $\theta=45^{\circ}$ to $51^{\circ} .\left\langle C_{T}\right\rangle_{T}$ without the end plate is the largest in the most range of $0^{\circ}<\theta \leq 100^{\circ}$. This contributes that $C_{T}$ without the end plate is the largest in the low tip speed ratio range. For $\gamma>0,\left\langle C_{T}\right\rangle_{T}$ takes the maximum value $\left(\left\langle C_{T}\right\rangle_{T}\right)_{\max }$ in all conditions, when the reference blade is in the range of $\theta=161^{\circ}$ to $167^{\circ}$, and the other blade is in the range of $\theta=341^{\circ}$ to $347^{\circ}$ near the most upstream. $\left(\left\langle C_{T}\right\rangle_{T}\right)_{\max }$ is decreased by attaching end plates and is the smallest at $\gamma=+45^{\circ} .\left\langle C_{T}\right\rangle_{T}$ without the end plate is largest in the most range of $45^{\circ} \leq \theta<100^{\circ}$ and $150^{\circ} \leq \theta \leq 165^{\circ}$. On the other hand, $\left\langle C_{T}\right\rangle_{T}$ takes the minimum value $\left(\left\langle C_{T}\right\rangle_{T}\right)_{\min }$ in the range of $\theta=45^{\circ}$ to $51^{\circ}$. It contributes to the largest $C_{T}$ without the end plate that $\left(\left\langle C_{T}\right\rangle_{T}\right)_{\max }$ without the end plate generated by one blade near the most upstream is the largest and $\left\langle C_{T}\right\rangle_{T}$ without the end plate is the largest in the most range of $45^{\circ} \leq \theta<100^{\circ}$ and $150^{\circ} \leq \theta \leq 165^{\circ}$. 
Next, for $\lambda=1.34,\left\langle C_{T}\right\rangle_{T}$ for $\gamma<0$ takes the maximum value $\left(\left\langle C_{T}\right\rangle_{T}\right)_{\max }$ in all conditions when the reference blade is at $\theta \cong 9^{\circ}$ near the most upstream. $\left(\left\langle C_{T}\right\rangle_{T}\right)_{\max }$ is increased by attaching end plates and is significantly large at $=-90^{\circ} .\left\langle C_{T}\right\rangle_{T}$ at $\gamma=-90^{\circ}$ is the largest in the range of $0^{\circ} \leq \theta<65^{\circ}$ which is over wide range around $\left(\left\langle C_{T}\right\rangle_{T}\right)_{\max }$. On the other hand $\left\langle C_{T}\right\rangle_{T}$ takes the minimum value $\left(\left\langle C_{T}\right\rangle_{T}\right)_{\min }$ in the range of $\theta=99^{\circ}$ to $105^{\circ}$. $\left(\left\langle C_{T}\right\rangle_{T}\right)_{\min }$ at $\gamma=-45^{\circ}$ is the largest and $\left(\left\langle C_{T}\right\rangle_{T}\right)_{\min }$ at $\gamma=-90^{\circ}$ is the smallest. Their difference is smaller than the difference of $\left(\left\langle C_{T}\right\rangle_{T}\right)_{\max }$. From the above, increasing in $\left(\left\langle C_{T}\right\rangle_{T}\right)_{\max }$ when the reference blade is at $\theta \cong 9^{\circ}$ near the most upstream contributes that $C_{T}$ and $\left(C_{T}\right)_{\max }$ are increased by attaching end plates in high tip speed ratio side and $\left(C_{T}\right)_{\max }$ becomes the largest at $\gamma=-90^{\circ}$. For $\gamma>0$, as for $\gamma<0,\left\langle C_{T}\right\rangle_{T}$ takes the maximum value $\left(\left\langle C_{T}\right\rangle_{T}\right)_{\max }$ at the phase where the reference blade is in the range of $\theta=9^{\circ}$ to $15^{\circ}$ near the most upstream in all conditions. However their differences by conditions are smaller than the differences for $\gamma<0$. In detail, $\left(\left\langle C_{T}\right\rangle_{T}\right)_{\max }$ without the end plate is the largest. $\left(\left\langle C_{T}\right\rangle_{T}\right)_{\min }$ at the phase where the reference blade is in the range of $\theta=99^{\circ}$ to $105^{\circ}$ is remarkably large at $\gamma=+45^{\circ}$, and the other two are comparable in magnitude. $\left\langle C_{T}\right\rangle_{T}$ at $\gamma=+45^{\circ}$ is remarkably large in the relatively wide range of

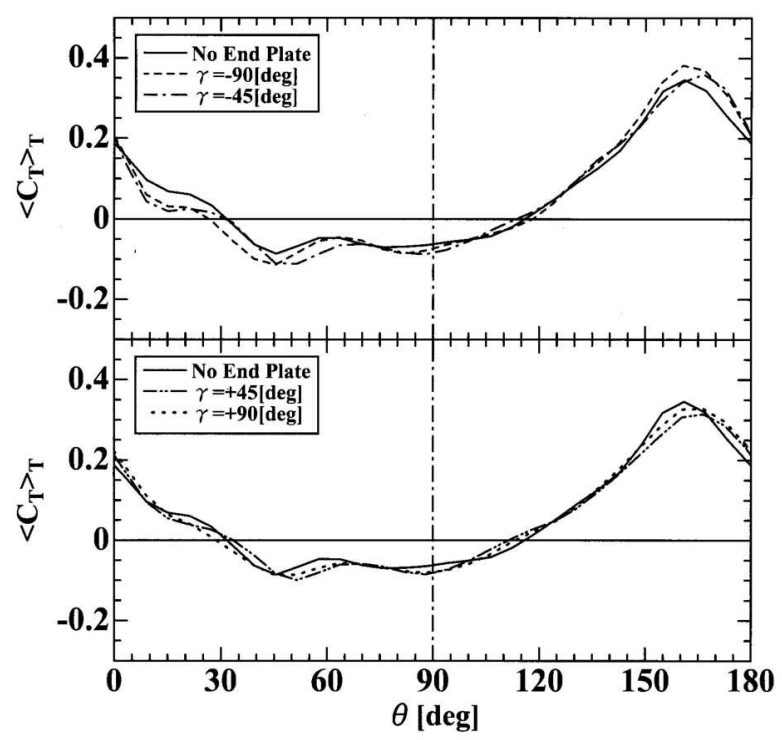

(a) $\lambda=0.63$

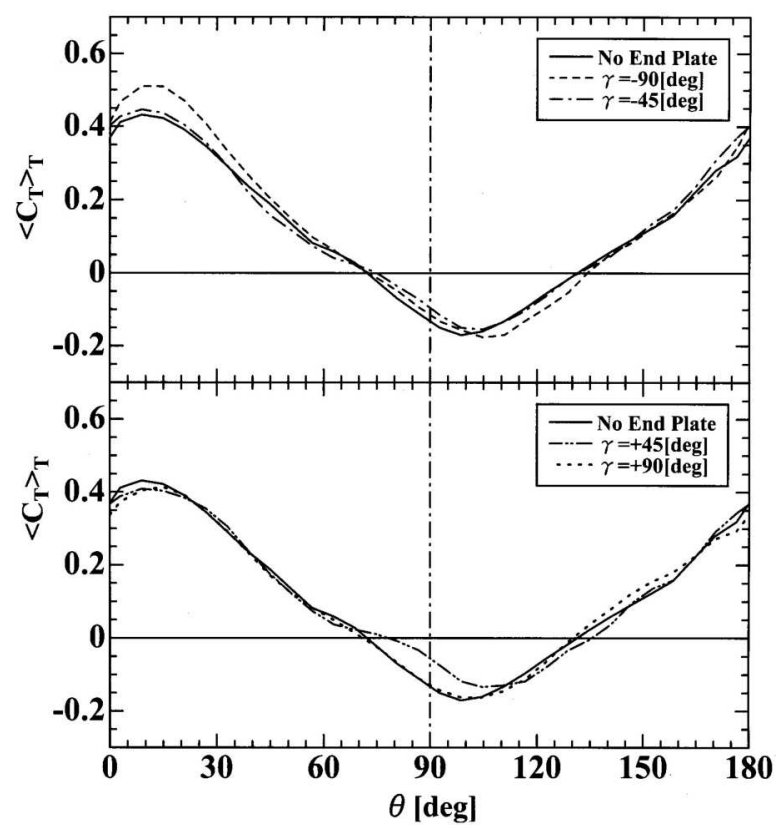

(b) $\lambda=1.34$

Fig.5 Effect of end plates on torque variation (two-blade wind turbine). 


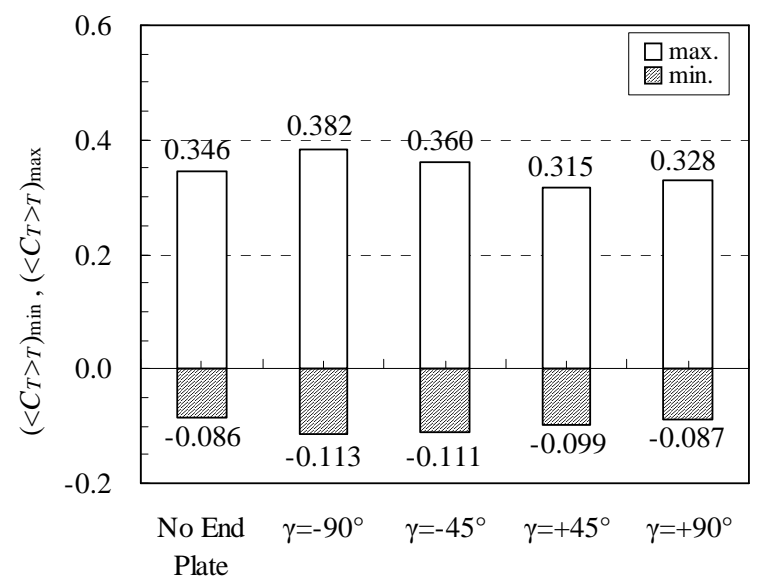

(a) $\lambda=0.63$

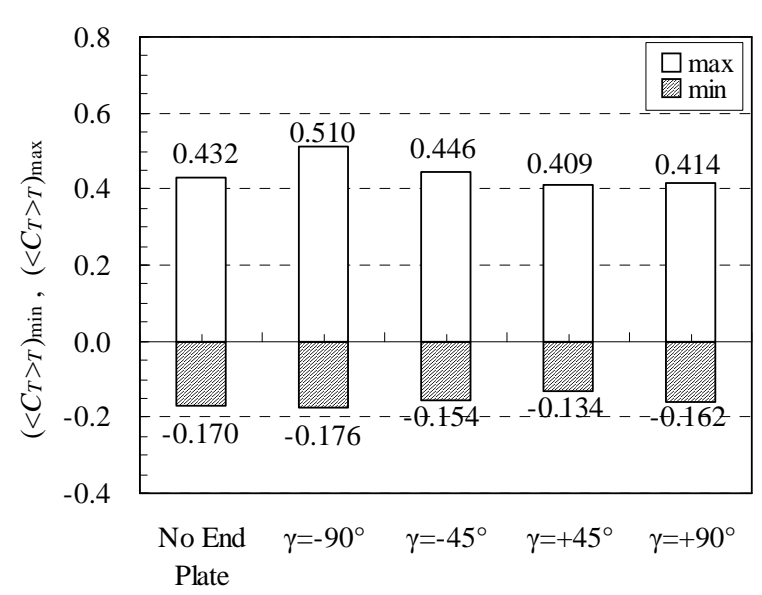

(b) $\lambda=1.34$

Fig. 6 The maximum and the minimum phase average torque coefficients (two-blade wind turbine).

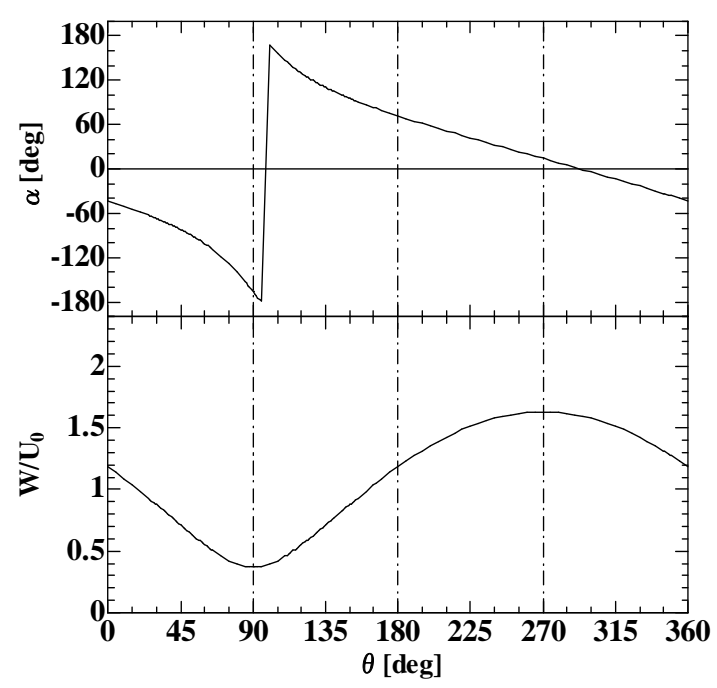

(a) $\lambda=0.63$

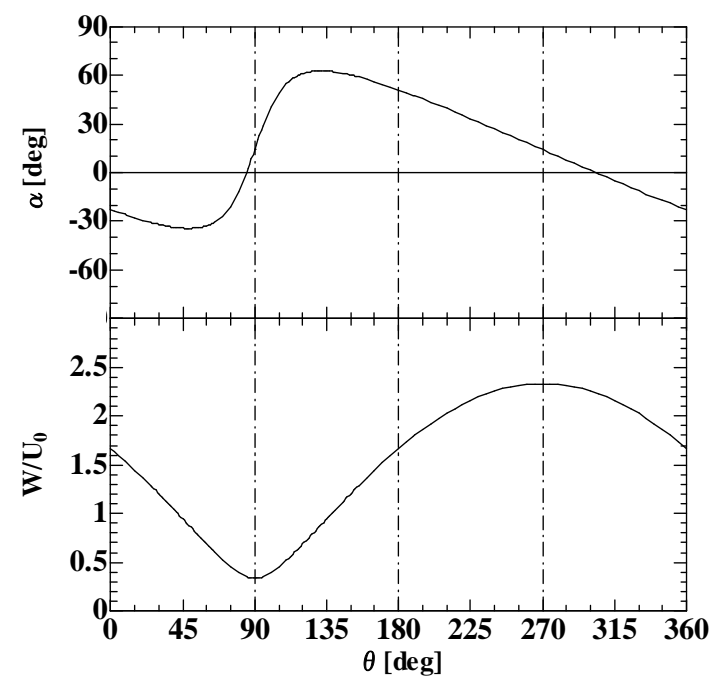

(b) $\lambda=1.34$

Fig.7 The angle of attack $\alpha$ and the relative velocity $W / U_{0}$ of each phase with respect to the reference blade.

$70^{\circ}<\theta<110^{\circ}$. This contributes to the large $C_{T}$ at $\gamma=+45^{\circ}$ compared with without the end plate. The trends of torque variation of without the end plate and $\gamma=+90^{\circ}$ are similar, but $C_{T}$ at $\gamma=+90^{\circ}$ is slightly smaller than without the end plate. Because $\left(\left\langle C_{T}\right\rangle_{T}\right)_{\max }$ at $\gamma=+90^{\circ}$ is slightly smaller than $\left(\left\langle C_{T}\right\rangle_{T}\right)_{\max }$ without the end plate.

\subsubsection{Torque variation of the single-blade wind turbine}

It is difficult to directly compare characteristics of a single-blade wind turbine and a two-blade wind turbine because their solidities are different. However in interpreting the torque fluctuation of the two-blade wind turbine, in order to investigate the contribution of each blade to the rotational torque, the torque variation of the single-blade wind turbine was examined. It is described in Section 5.2.3 that the characteristics of the torque variation of the two-blade wind turbine can be qualitatively interpreted from the torque variation of the single-blade wind turbine.

Figure 8 (a) and (b) show torque variation as a function of the azimuth angle $\theta$ of the wind turbine with a single blade having four types of end plates with four different setting angles and without the end plate as shown in Fig. 5. Figure 9 (a) and (b) show the maximum and minimum phase average torque coefficients of these five types of wind turbines with a single blade. Typically, cases of $\lambda=0.63$ and $\lambda=1.34$ are shown as well as the wind turbine with two blades. For $\lambda=0.63,\left\langle C_{T}\right\rangle_{T}$ for $\gamma<0$ takes the maximum value $\left(\left\langle C_{T}\right\rangle_{T}\right)_{\max }$ in all conditions when the reference blade is in range of $\theta=336^{\circ}$ to $337^{\circ}$ near the most upstream. $\left(\left\langle C_{T}\right\rangle_{T}\right)_{\max }$ is increased by attaching end plates and is the largest at $\gamma=-90^{\circ}$. These trends correspond to $\left(\left\langle C_{T}\right\rangle_{T}\right)_{\max }$ trends of the two-blade wind turbine. $\left\langle C_{T}\right\rangle_{T}$ without the end plate is larger than the others in the most range of $0^{\circ}<\theta<75^{\circ}$ where the blade moves from the upstream side to 
the downstream side. This corresponds to the fact that $\left\langle C_{T}\right\rangle_{T}$ without the end plate is larger in the majority region of $0^{\circ}<\theta \leq 100^{\circ}$ in the two-blade wind turbine compared with the others. This contributes to the largest $C_{T}$ without the end plate in the low tip speed ratio range. Here, it is considered that $\left\langle C_{T}\right\rangle_{T}$ without the end plate is larger than the others in the range of $0^{\circ}<\theta<75^{\circ}$. Assuming that the wind velocity is uniform for $\lambda<1$, the angle of attack $\alpha<0$ to the relative wind velocity (the rotating shaft side is the negative pressure surface) monotonically decreases from $\alpha>$ $-90^{\circ}$ to $\alpha<-90^{\circ}$ in this range. In the range of $\alpha>-90^{\circ}$, the lift contributes to the torque in the rotating direction of the wind turbine, and the drag contributes to the torque in the direction opposite to the rotation of the wind turbine. On the other hand, in the range of $\alpha<-90^{\circ}$, the lift contributes to the torque in the direction opposite to the rotation, and the drag contributes to the torque in the rotating direction. In this region, a large-scale vortex is generated near the wing tip of the three-dimensional blade without the end plate (Ferreira, C. et al., 2006), consequently the low-pressure region of the vortex contributes to the rotational torque as lift in the region of $\alpha>-90^{\circ}$, and as drag in the region of $\alpha<-90^{\circ}$. When end plates are attached, the generation of this vortex is suppressed, it is expected that $\left\langle C_{T}\right\rangle_{T}$ without the end plate becomes larger. $\left\langle C_{T}\right\rangle_{T}$ takes the minimum value $\left(\left\langle C_{T}\right\rangle_{T}\right)_{\min }$ in the range of $\theta=227^{\circ}$ to $228^{\circ}$. $\left(\left\langle C_{T}\right\rangle_{T}\right)_{\min }$ at $\gamma=-90^{\circ}$ is the smallest, and the other two are comparable in magnitude. The differences in $\left(\left\langle C_{T}\right\rangle_{T}\right)_{\min }$ by conditions are smaller than the differences in $\left(\left\langle C_{T}\right\rangle_{T}\right)_{\max }$. In all conditions, $\left\langle C_{T}\right\rangle_{T}$ takes a maximal value around $\theta=170^{\circ}$, it is the largest without the end plate and is the smallest at $\gamma=-45^{\circ}$. In all conditions, $\left\langle C_{T}\right\rangle_{T}$ takes a minimal value around $\theta=30^{\circ}$, it is significantly large without the end plate and is the smallest at $\gamma=-90^{\circ} .\left\langle C_{T}\right\rangle_{T}$ for $\gamma>0$ takes the maximum value $\left(\left\langle C_{T}\right\rangle_{T}\right)_{\max }$ in all conditions, when the reference blade is in the range of $\theta=336^{\circ}$ to $337^{\circ}$ near the most upstream. $\left(\left\langle C_{T}\right\rangle_{T}\right)_{\max }$ is the largest at $\gamma=+90^{\circ}$ and is the smallest at $\gamma=+45^{\circ}$. The differences in $\left(\left\langle C_{T}\right\rangle_{T}\right)_{\max }$ by conditions are smaller than the differences for $\gamma<0$. In all conditions, $\left\langle C_{T}\right\rangle_{T}$ takes the maximal value near $\theta=170^{\circ}$, and it is the largest without the end plate. This contributes to the largest $\left(\left\langle C_{T}\right\rangle_{T}\right)_{\max }$ without the end plate taken near the most upstream in the two-blade wind turbine. This is because in the two-blade wind turbine, when one blade is in the vicinity of $\theta=170^{\circ}$, the other blade is in the vicinity of $\theta=350^{\circ}$. In the most region of $57^{\circ}<\theta<105^{\circ}$ and in the region of $150^{\circ}<\theta<180^{\circ},\left\langle C_{T}\right\rangle_{T}$ is the largest without the end plate and the others are comparable in magnitude. These correspond to that $\left\langle C_{T}\right\rangle_{T}$ of the two-blade wind turbine is the largest without the end plate in the most region of $45^{\circ}<\theta<100^{\circ}$ and in the region of $150^{\circ}<\theta<165^{\circ} .\left\langle C_{T}\right\rangle_{T}$ takes the minimum value $\left(\left\langle C_{T}\right\rangle_{T}\right)_{\text {min }}$ in the range of $\theta=227^{\circ}$ to $228^{\circ} .\left(\left\langle C_{T}\right\rangle_{T}\right)_{\min }$ at $\gamma=+90^{\circ}$ is the smallest, and $\left(\left\langle C_{T}\right\rangle_{T}\right)_{\min }$ at $\gamma=+45^{\circ}$ is the largest. $\left\langle C_{T}\right\rangle_{T}$ takes minimal value in the range of $\theta=30^{\circ}$ to $40^{\circ}$, the minimal value of $\left\langle C_{T}\right\rangle_{T}$ in the range of $\theta=30^{\circ}$ to $40^{\circ}$ is the smallest at $\gamma=+45^{\circ}$, and the other two are comparable in magnitude. The differences in the minimal value of $\left\langle C_{T}\right\rangle_{T}$ for $\gamma>0$ are smaller than the differences in the minimal value of $\left\langle C_{T}\right\rangle_{T}$ taken around $\theta=30^{\circ}$ for $\gamma<0$. The tendency of this minimal value corresponds to that $\left(\left\langle C_{T}\right\rangle_{T}\right)_{\min }$ of the two-blade wind turbine taken in the range of $\theta=45^{\circ}$ to $51^{\circ}$ is the smallest at $\gamma=+45^{\circ}$ and the other two are comparable in magnitude.

For $\lambda=1.34,\left\langle C_{T}\right\rangle_{T}$ for $\gamma<0$ takes the maximum value $\left(\left\langle C_{T}\right\rangle_{T}\right)_{\max }$ in all conditions, when the reference blade is at $\theta \cong 2^{\circ}$ near the most upstream. It is increased by attaching end plates and is the largest at $\gamma=-90^{\circ}$. These trends correspond to that $\left\langle C_{T}\right\rangle_{T}$ of the two-blade wind turbine at $\theta \cong 9^{\circ}$ near the most upstream takes the maximum value $\left(\left\langle C_{T}\right\rangle_{T}\right)_{\max }$, it is increased by attaching end plates and $\left(\left\langle C_{T}\right\rangle_{T}\right)_{\max }$ is remarkably large at $\gamma=-90^{\circ}$. $\left\langle C_{T}\right\rangle_{T}$ takes two minimal values near $\theta=95^{\circ}$ and $\theta=270^{\circ}$, and one of them is the minimum value. The minimal value near $\theta=95^{\circ}$ is the largest at $\gamma=-45^{\circ}$ and is the smallest at $\gamma=-90^{\circ}$, but their differences are small. The minimal value near $\theta=$ $270^{\circ}$ is remarkably large at $\gamma=+45^{\circ}$ and the other two are comparable in magnitude. These results correspond to the fact that $\left(\left\langle C_{T}\right\rangle_{T}\right)_{\min }$ taken in the range of $\theta=99^{\circ}$ to $105^{\circ}$ in the two-blade wind turbine is the largest at $\gamma=-45^{\circ}$ and is the smallest at $\gamma=-90^{\circ}$. For $\gamma>0,\left\langle C_{T}\right\rangle_{T}$ takes the maximum value $\left(\left\langle C_{T}\right\rangle_{T}\right)_{\max }$ in the range of $\theta=2^{\circ}$ to $9^{\circ}$ near the most upstream in all conditions. $\left(\left\langle C_{T}\right\rangle_{T}\right)_{\max }$ becomes slightly larger by attaching end plates, but their differences are smaller than the differences for $\gamma<0$. In detail, $\left(\left\langle C_{T}\right\rangle_{T}\right)_{\max }$ at $\gamma=+90^{\circ}$ is slightly larger than the others. Similar to the case of $\gamma<0,\left\langle C_{T}\right\rangle_{T}$ takes the minimal value at two phases of $\theta=95^{\circ}$ and $=270^{\circ}$, and either one takes the minimum value $\left(\left\langle C_{T}\right\rangle_{T}\right)_{\min }$. These minimal values are the largest at $\gamma=+45^{\circ}$ and the other two are comparable in magnitude. In the region around these two minimal values, $\left\langle C_{T}\right\rangle_{T}$ at $\gamma=+45^{\circ}$ is larger in relatively wide ranges of $75^{\circ}<\theta<112^{\circ}$ in which the blade moves from the upstream side to the downstream side passing $\theta=90^{\circ}$, and the region $236^{\circ}<\theta<315^{\circ}$ in which the blade moves from the downstream side to the upstream side passing $\theta=270^{\circ}$. These facts concerning the two minimal values correspond to the fact that $\left(\left\langle C_{T}\right\rangle_{T}\right)_{\min }$ of the two-blade wind turbine taken when the reference blade is in the range of $\theta=99^{\circ}$ to $105^{\circ}$ (the other blade is in the range of $\theta=279^{\circ}$ to $285^{\circ}$ ) is remarkably large at $\gamma=+45^{\circ}$ and the other two are comparable in magnitude, and $\left\langle C_{T}\right\rangle_{T}$ at $\gamma=+45^{\circ}$ is remarkably 
larger than the others, when the reference blade is in the relatively wide range of $70^{\circ}<\theta<110^{\circ}$ (the other blade is in the range of $\theta=250^{\circ}$ to $290^{\circ}$ ).

Here, at $\lambda=1.34,\left(\left\langle C_{T}\right\rangle_{T}\right)_{\max }$ taken near the most upstream and $\left(\left\langle C_{T}\right\rangle_{T}\right)_{\min }$ taken at $\theta=95^{\circ}$ or $\theta=270^{\circ}$ are considered from the view point of flow field. First, $\left(\left\langle C_{T}\right\rangle_{T}\right)_{\max }$ is considered. In the region where the blade moves from near the most upstream side to the downstream side, dynamic stalling phenomenon occurs due to generation and passage of a large-scale vortex over the entire suction surface (the surface on the rotation axis side), and large lift is generated in the blade (Paraschivoiu, I., translated by Hayashi et al. 2007). On the other hand, since the tip vortex is generated in the three-dimensional blade (Furuya, Y. 1973), when the blade of the wind turbine is in the dynamic stall state, it is considered that this tip vortex influences the large-scale vortex and contributes to the reduction of the lift force. Therefore, it is considered that strength of the tip vortex is suppressed by attaching the end plate, and reduction in lift is reduced, so $\left(\left\langle C_{T}\right\rangle_{T}\right)_{\max }$ with end plates becomes larger. The extent of the increase of $\left(\left\langle C_{T}\right\rangle_{T}\right)_{\max }$ is large in the order of $\gamma=-90^{\circ}, \gamma=-45^{\circ}, \gamma=+90^{\circ}, \gamma=+45^{\circ}$ from the experimental results. Next, $\left(\left\langle C_{T}\right\rangle_{T}\right)_{\min }$ is taken at $\theta=95^{\circ}$ or $\theta=270^{\circ}$, the contribution of drag to the wind turbine rotation torque is large around these phases. In this region, provided that the wind uniformly flows into the wind turbine, the angle of attack against the relative wind velocity varies considerably between positive and negative across $\alpha=0^{\circ}$. In this case, it is considered that the influence of

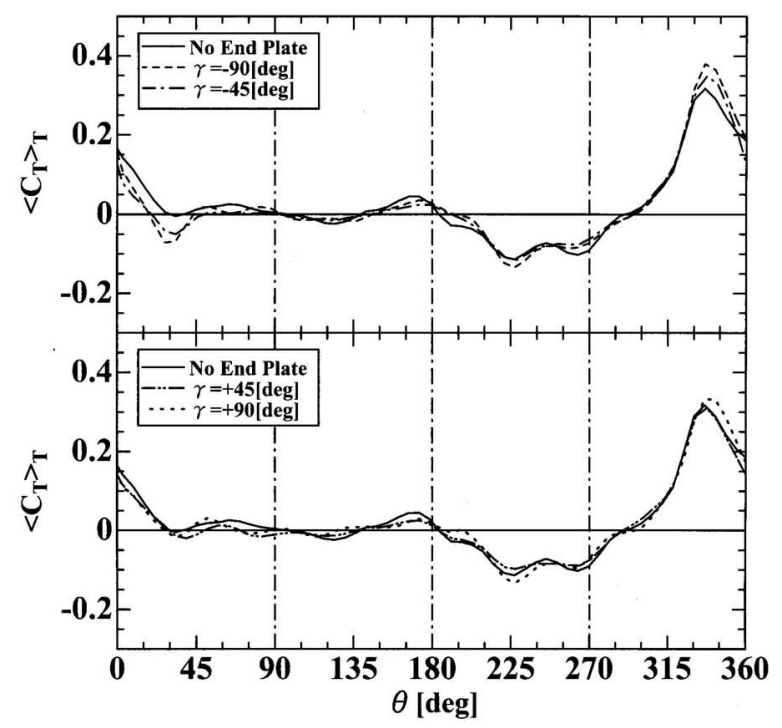

(a) $\lambda=0.63$

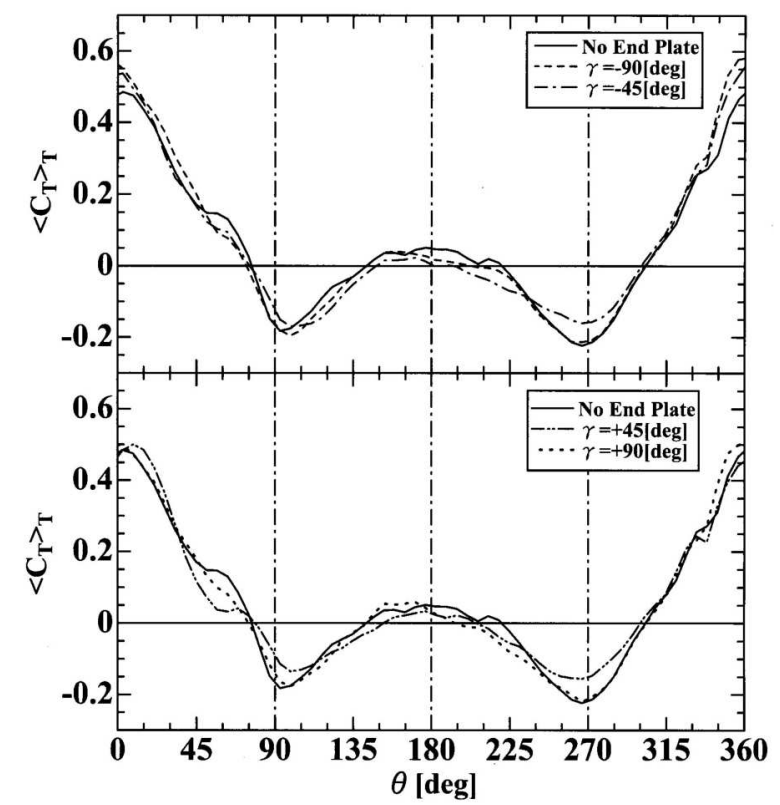

(b) $\lambda=1.34$

Fig.8 Effect of end plates on torque variation (single-blade wind turbine). 


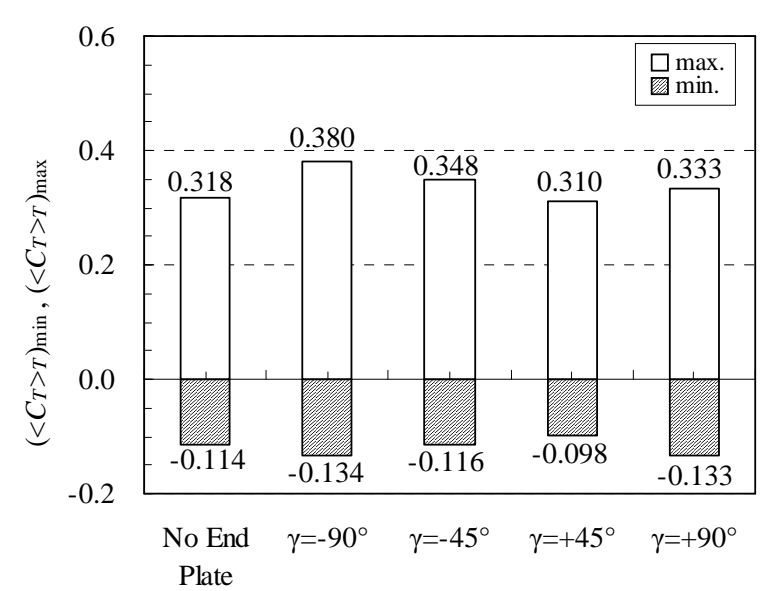

(a) $\lambda=0.63$

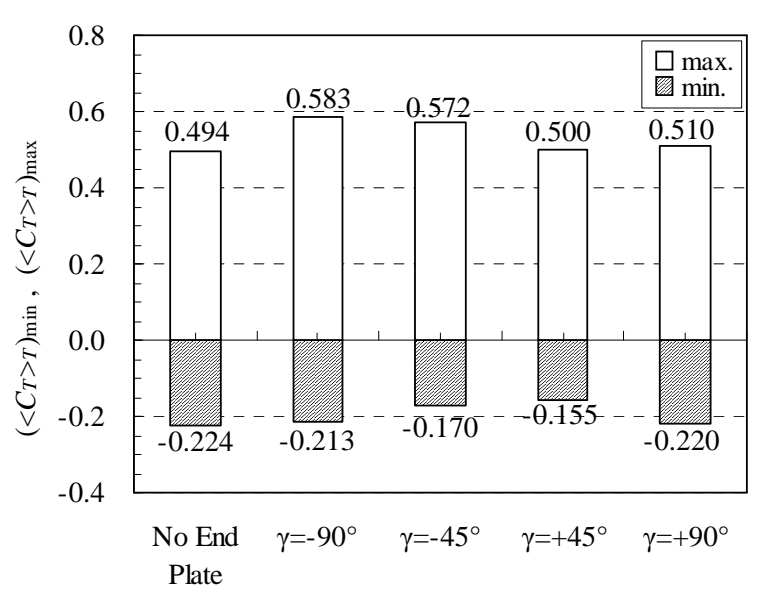

(b) $\lambda=1.34$

Fig.9 The maximum and the minimum phase average torque coefficients (single-blade wind turbine).

form drag and induced drag of the blade is large as drag force. It is expected that the drag reduction effect is greater at $\gamma= \pm 45^{\circ}$ than at $\gamma= \pm 90^{\circ}$.

\subsubsection{Two-blade wind turbine torque variation calculated from single-blade wind turbine torque variation}

It is difficult to directly compare characteristics of a single-blade wind turbine and a two-blade wind turbine because their solidities are different, however the torque variation characteristics of the two-blade wind turbine will be studied based on the torque variation characteristics of the single-blade wind turbine. Therefore, using the torque variation data of the single-blade wind turbine, the torque variation of the wind turbine having two blades attached with the phase difference of $180^{\circ}$ is calculated. In detail, the torque variation of the single-blade wind turbine is divided into regions of $\theta=0^{\circ}$ to $180^{\circ}$ and $\theta=180^{\circ}$ to $360^{\circ}$, the phase of the torque variation of $\theta=180^{\circ}$ to $360^{\circ}$ is shifted by $180^{\circ}$, the torque variation is replaced with the torque variation of the region of $\theta=0^{\circ}$ to $180^{\circ}$, and by adding this to the original torque variation of the range of $\theta=0^{\circ}$ to $180^{\circ}$, the torque variation of the two-blade wind turbine is calculated. At this time, the torque variation of the single-blade wind turbine was measured by removing only one blade while leaving the arms in Fig. 3. Figures 10 (a) and (b) show the torque variation of the two-blade wind turbine calculated from the torque variation of the single-blade wind turbine at $\lambda=0.63$ and $\lambda=1.34$, respectively. For $\lambda=0.63,\left\langle C_{T}\right\rangle_{T}$ for $\gamma<0$ takes the maximum value $\left(\left\langle C_{T}\right\rangle_{T}\right)_{\max }$ in all conditions, when the reference blade is in the range of $\theta=154^{\circ}$ to $155^{\circ}$, and the other blade is in the range of $\theta=334^{\circ}$ to $335^{\circ}$ near the most upstream. $\left(\left\langle C_{T}\right\rangle_{T}\right)_{\max }$ is increased by attaching end plates and is the largest at $\gamma=-90^{\circ} .\left\langle C_{T}\right\rangle_{T}$ takes the minimum value $\left(\left\langle C_{T}\right\rangle_{T}\right)_{\min }$ in the range of $\theta=33^{\circ}$ to $40^{\circ}$, and it is the largest without the end plate. In the wide range of $0^{\circ}<\theta<70^{\circ},\left\langle C_{T}\right\rangle_{T}$ without the end plate is larger than that of the other two. The above tendency is similar to the tendency of the torque variation of the two-blade wind turbine shown on the upper side of Fig. 5 (a). However, the values of the phase and phase average torque coefficients are different. $\left\langle C_{T}\right\rangle_{T}$ for $\gamma>0$ takes the maximum value $\left(\left\langle C_{T}\right\rangle_{T}\right)_{\max }$ in all conditions, when the reference blade is at $\theta=155^{\circ}$, and the other blade is at $\theta=335^{\circ}$ near the most upstream. $\left(\left\langle C_{T}\right\rangle_{T}\right)_{\max }$ is about the same regardless of the presence or absence of the end plate. In the range of $0^{\circ}<\theta<8^{\circ}, 55^{\circ}<\theta<80^{\circ}$ and $172^{\circ}<\theta<180^{\circ}$, $\left\langle C_{T}\right\rangle_{T}$ is the largest without the end plate. $\left\langle C_{T}\right\rangle_{T}$ takes the minimum value $\left(\left\langle C_{T}\right\rangle_{T}\right)_{\min }$ in the range of $\theta=39^{\circ}$ to $40^{\circ},\left(\left\langle C_{T}\right\rangle_{T}\right)_{\min }$ is the smallest at $\gamma=+90^{\circ}$, and the other two are comparable in magnitude. In all conditions, the tendency that $\left\langle C_{T}\right\rangle_{T}$ takes the maximum value $\left(\left\langle C_{T}\right\rangle_{T}\right)_{\max }$ when one of the blades is near the most upstream, $\left\langle C_{T}\right\rangle_{T}$ is the largest without the end plate in the range of $55^{\circ}<\theta<80^{\circ}$, and $\left\langle C_{T}\right\rangle_{T}$ takes the minimum value $\left(\left\langle C_{T}\right\rangle_{T}\right)_{\min }$ in the range of $\theta=39^{\circ}$ to $40^{\circ}$ is similar to the tendency of the torque variation of the two-blade wind turbine shown in the lower side of Fig. 5 (a), although the values of the phase and phase average torque coefficient are different.

For $\lambda=1.34,\left\langle C_{T}\right\rangle_{T}$ for $\gamma<0$ takes the maximum value $\left(\left\langle C_{T}\right\rangle_{T}\right)_{\max }$ when the reference blade is in the range of $3^{\circ}<\theta<9^{\circ}$ or at $\theta=177^{\circ}$, that is, one of the blades is near the most upstream in all conditions. $\left(\left\langle C_{T}\right\rangle_{T}\right)_{\max }$ is increased by attaching end plates and is the largest at $\gamma=-90^{\circ}$. In most range of $0^{\circ}<\theta<48^{\circ}$ and $135^{\circ}<\theta<180^{\circ}$ around $\left(\left\langle C_{T}\right\rangle_{T}\right)_{\max },\left\langle C_{T}\right\rangle_{T}$ at $\gamma=-90^{\circ}$ is larger than that of the other two. $\left\langle C_{T}\right\rangle_{T}$ takes the minimum value $\left(\left\langle C_{T}\right\rangle_{T}\right)_{\min }$ in the range of $\theta=93^{\circ}$ to $99^{\circ},\left(\left\langle C_{T}\right\rangle_{T}\right)_{\min }$ is the smallest at $\gamma=-45^{\circ}$, and the other two are comparable in magnitude. In the relatively wide range of $67^{\circ}<\theta<109^{\circ},\left\langle C_{T}\right\rangle_{T}$ at $\gamma=-45^{\circ}$ is larger than that of the other two. In all conditions, the 
tendency that $\left\langle C_{T}\right\rangle_{T}$ takes $\left(\left\langle C_{T}\right\rangle_{T}\right)_{\max }$ when one of the blades is near the most upstream, and $\left(\left\langle C_{T}\right\rangle_{T}\right)_{\max }$ is increased by attaching end plates and is largest at $\gamma=-90^{\circ}$, in most range of $0^{\circ}<\theta<48^{\circ}$ and $135^{\circ}<\theta<180^{\circ}$ around $\left(\left\langle C_{T}\right\rangle_{T}\right)_{\max },\left\langle C_{T}\right\rangle_{T}$ at $\gamma=-90^{\circ}$ is larger than that of the other two, and $\left\langle C_{T}\right\rangle_{T}$ takes $\left(\left\langle C_{T}\right\rangle_{T}\right)_{\min }$ in the range of $\theta=93^{\circ}$ to $99^{\circ}$ is similar to the tendency of the torque variation of the two-blade wind turbine shown in the upper side of Fig. 5 (b). However, the values of the phase and phase average torque coefficient are different. $\left\langle C_{T}\right\rangle_{T}$ for $\gamma<0$ takes the maximum value $\left(\left\langle C_{T}\right\rangle_{T}\right)_{\max }$ when the reference blade is in the range of $3^{\circ}<\theta<9^{\circ}$ or at $\theta=177^{\circ}$, that is, one of blades is near the most upstream in all conditions. However, the differences by conditions are small. In addition, $\left\langle C_{T}\right\rangle_{T}$ takes the minimum value $\left(\left\langle C_{T}\right\rangle_{T}\right)_{\min }$ at $\theta=93^{\circ},\left(\left\langle C_{T}\right\rangle_{T}\right)_{\min }$ is remarkably large at $\gamma=+45^{\circ}$, and the other two are comparable in magnitude. $\left\langle C_{T}\right\rangle_{T}$ at $\gamma=+45^{\circ}$ in the relatively wide range of $70^{\circ}<\theta<126^{\circ}$ is remarkably larger than that of the other two. The tendency of these torque variation is similar to the tendency of the torque variation of the two-blade wind turbine shown on the lower side of Fig. 5 (b), although its value and the phase are different.

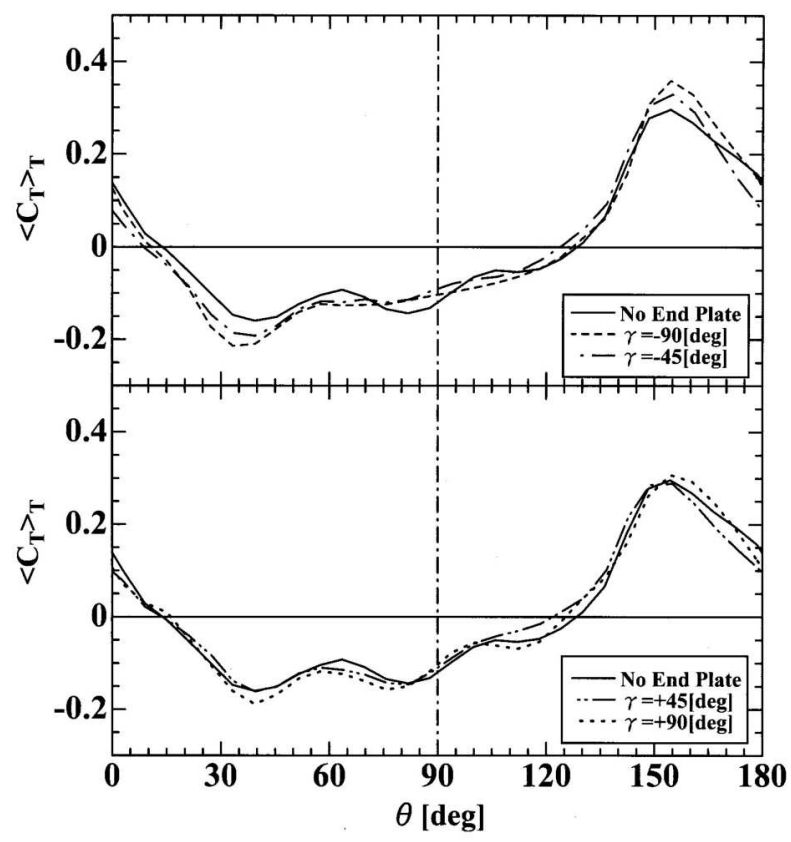

(a) $\lambda=0.63$

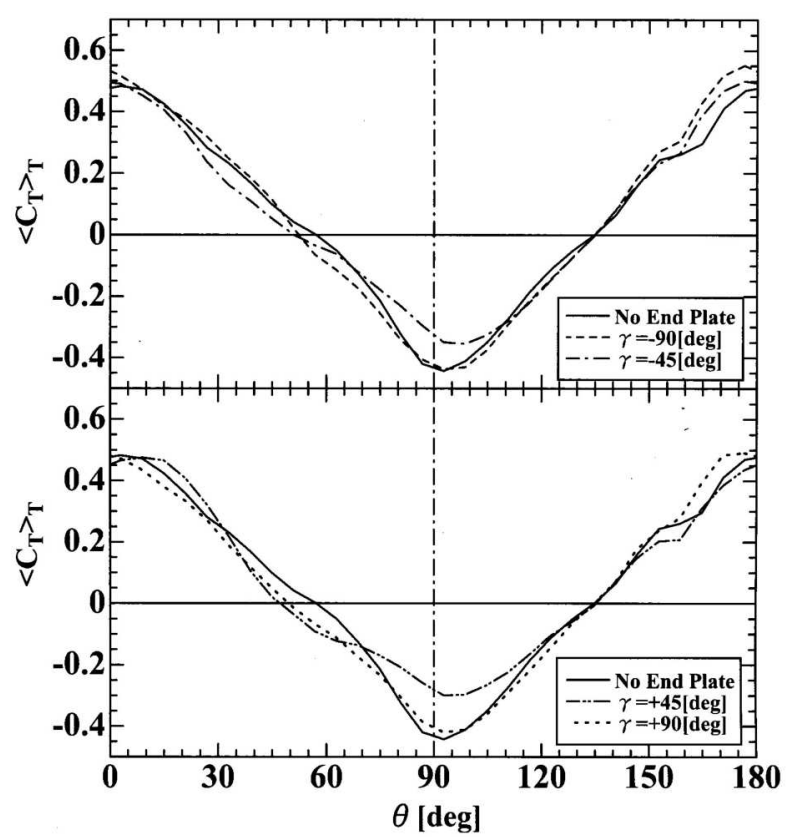

(b) $\lambda=1.34$

Fig.10 Torque variation of the two-blade wind turbine calculated from torque variation of the single-blade wind turbine. 
From the above, the torque variation of the two-blade wind turbine calculated from the torque variation data of the single-blade wind turbine is qualitatively similar to the torque variation of the two-blade wind turbine in both $\lambda=0.63$ and 1.34. Therefore, it shows that it is possible to interpret the tendency of the torque variation of the two-blade wind turbine from the torque variation of the single-blade wind turbine.

\section{Conclusions}

(1) In the low tip speed ratio range of $\lambda<0.87, C_{T}$ and $C_{P}$ are reduced by attaching end plates.

(2) In the high tip speed ratio range of $\lambda>1.18, C_{T}$ and $C_{P}$ are increased by attaching end plates except for $\gamma=+90^{\circ}$, and $\left(C_{T}\right)_{\max }$ and $\left(C_{P}\right)_{\max }$ are the largest at $\gamma=-90^{\circ}$.

(3) $C_{T}$ without the end plate in the low tip speed ratio range is the largest because $\left\langle C_{T}\right\rangle_{T}$ without the end plate increases in the range of $0^{\circ}<\theta<75^{\circ}$ where the blade moves from the most upstream side to the downstream side.

(4) The reasons why $C_{T}$ is increased by attaching end plates except for $\gamma=+90^{\circ}$ in the high tip speed ratio side are that $\left(\left\langle C_{T}\right\rangle_{T}\right)_{\max }$ for $\gamma<0$ taken near the most upstream is increased, and $\left\langle C_{T}\right\rangle_{T}$ for $\gamma>0$ at $\gamma=+45^{\circ}$ is increased in a relatively wide range around two minimal values of $\left\langle C_{T}\right\rangle_{T}$ taken near $\theta=90^{\circ}$ and $270^{\circ}$.

\section{Acknowledgments}

In the wind tunnel experiment, we received great cooperation from student trainee Mr. Naoki Nishikawa (then Yamaguchi University graduate student). We thank him very much.

\section{References}

Asao, T., Iida. K., Kimura, Y., Sakuyama, T., Tani, K., Horiuchi, K. and Seki, K., Aerodynamic performance of vertical axis wind turbine in low circumferential speed ratio, Proceedings of the 31th Symposium on Wind Energy (2009), pp.325-328 (in Japanese).

Ferreira, C., Van Kuik, G. and Van Bussel, G., Wind turbine hotwire measurements, flow visualization and thrust measurement of a VAWT in skew, 44 ${ }^{\text {th }}$ AIAA Aerospace Sciences Meeting and Exhibit 9-12 January (2006), pp.1-12.

Furuya, Y., Fluid dynamics <Basic course> (1973), pp.113-121, Kyouritsu publishing (in Japanese).

Hashidate, M. and Ishida, Y., Wall interference correction of an aerodynamic efficiency of a windmill in wind tunnel test, Journal of Japan Wind Energy Association, Vol.15, No.1(1991), pp.21-27(in Japanese).

Hofemann, C., Simao Ferreira, C., Van Bussel, G., Van Kuik, G., Scarano, F. and Dixon, K., 3D stereo PIV study of tip vortex evolution on a VAWT, European Wind Energy Association EWEA, (2008), pp.1-8.

Kubota, H., Arai, I., Matsuzaka, M., Koshioka, Y. and Kawakatsu, H., Wind tunnel experiment on effect of the end plate at low wind speed, Journal of Japan Society for Aeronautical and Space Sciences, Vol.31, No.359 (1983), pp.684-689 (in Japanese).

Mizuno, A., The Scale Effect of Straight-Wing Vertical-Axis Wind Turbines, Proceedings of the 24th Symposium on Wind Energy (2002), pp.195-197 (in Japanese).

Mueller, T. J. and DeLaurier, J. D., Aerodynamics of small vehicles, Annual Review of Fluid Mechanics, Vol.35, (2003), pp.89-111.

Murotsu, Y. edited, Introduction to aerospace engineering [Second edition] (2006),pp.46-47, Morikita publishing (in Japanese).

Nishizawa, Y., Suzuki, M., Taniguchi, H. and Ushiyama, I., Experimental study of vertical axis type wind turbine, Transactions of the JSME, B, Vol.75, No.751(2009), pp.550-552(in Japanese).

Paraschivoiu, I., translated by Hayashi, T., Kawamura, T, Hara, Y. and Tagawa, K., Wind turbine design with emphasis on Darrieus concept (2007), pp.115-116, Index publishing (in Japanese).

Ushiyama, I., Introduction of the Wind Turbine Engineering (2002), p.55, Morikita publishing (in Japanese).

Yamada, S., Tamura, T. and Mochizuki, S., Effects of Wing Section on Mean Characteristics and Temporal Torque Variation for Small Straight-Bladed Vertical Axis Wind Turbine, Journal of Fluid Science and Technology, Vol.6, No.6 (2011), pp.875-886.

Yamada, S., Tamura, T. and Mochizuki, S., Effect of the Reynolds number on the performance and approximate modeling of the small straight-bladed vertical-axis wind turbine, Journal of Fluid Science and Technology, Vol.11, No.3,(2016), JFST0014. 